\title{
Displaced Fracture of the Proximal Humerus Causing Axillary Artery Injury and Brachial Plexus Palsy
}

\author{
Younus $\mathrm{A}^{1}$, Kelly $\mathrm{A}^{2^{*}}$ and Lekgwara $\mathrm{P}^{2}$ \\ ${ }^{1}$ Department of Orthopedics, Helen Joseph Hospital, University of the Witwatersrand, Johannesburg, South Africa \\ ${ }^{2}$ Department of Neurosurgery, Dr. George Mukhari Academic Hospital, Sefako Makgatho Health Sciences \\ University, Pretoria, South Africa
}

${ }^{*}$ Corresponding author: Kelly A, Department of Neurosurgery, Dr. George Mukhari Academic Hospital, Sefako Makgatho Health Sciences University, Pretoria, South Africa, Tel: +27 84677 3771, E-mail: adriankelly1000@yahoo.co.uk

Citation: Younus A, Kelly A, Lekgwara P (2018) Displaced Fracture of the Proximal Humerus Causing Axillary Artery Injury and Brachial Plexus Palsy. J Orthop Physiother 1(1): 104. doi: 10.15744/2639-930X.1.104

Received Date: August 07, 2018 Accepted Date: September 19, 2018 Published Date: September 21, 2018

\begin{abstract}
Introduction: Proximal humeral fractures account for approximately $5 \%$ of fractures seen in the emergency department. Despite this relatively high incidence only $15 \%$ of these fractures are displaced with an even smaller proportion being severely displaced enough to cause an axillary artery injury.

Materials and methods: We report a case of an elderly female patient who slipped and fell at home. She presented to our unit complaining of left shoulder pain and an inability to feel or move the involved limb. Imaging studies confirmed the presence of a severely displaced fracture of the proximal humerus and occlusion of the axillary artery. She was taken for open reduction and internal fixation of the proximal humerus followed by immediate axillary artery endovascular stenting.

Results: Post-operative clinical examination and vascular imaging studies confirmed good distal flow however in the immediate postoperative period the limb remained paralyzed. Brachial plexus palsy was subsequently confirmed to be a neuropraxia on nerve conduction studies. This was managed conservatively and at her three month out-patient review the patient was noted to have significantly improved power in the involved limb and was doing well.

Discussion: Displaced fractures of the proximal humerus causing an axillary artery injury and brachial plexus palsy are rarely seen. Due to tethering from the anterior and posterior circumflex arteries the axillary artery finds itself vulnerable to compression by a displaced humeral shaft. The proximal axillary artery and brachial plexus are contained within the same fascial sheath and while this affords protection it may also result in a brachial plexus palsy caused either by direct trauma from the fractured bone or due to oedema and/or compression from hematoma.

Conclusion: We report a rare case of a severely displaced fracture of the proximal humeral shaft that caused blunt trauma to the axillary artery and brachial plexus palsy. Fortunately the attending surgeons accurately diagnosed and managed the orthopedic, vascular and neural aspects of the injury and the case had a favorable outcome.
\end{abstract}

Keywords: Proximal Humeral Fracture; Axillary Artery Injury; Brachial Plexus Palsy.

List of Abbreviations: CT-Computed Tomography; CTA-Computed Tomographic Angiography.

\section{Introduction}

Fractures of the proximal humerus are relatively common and overall account for 4-5\% of fractures seen in a hospital emergency department [1]. Despite this relatively high incidence only $15 \%$ of these fractures are displaced and hence the concurrent occurrence of an axillary artery injury is rare. Due to the proximal axillary artery and brachial plexus sharing the same fascial sheath once an axillary artery occurs there may be associated brachial plexus palsy [2]. This brachial plexus palsy is caused either by direct trauma from the fractured bone, which is commonly blunt in nature, or due to oedema and/or compression from hematoma [3,4].We report a rare case of an elderly female, whom after a simple fall, suffered a severely displaced fracture of the proximal humeral shaft that caused blunt trauma to the axillary artery and brachial plexus palsy.

\section{Clinical Case}

We report a case of an elderly female patient in her 8th decade who slipped and fell at home. She presented acutely to our unit 
complaining of severe pain in her left shoulder and an inability to feel or move the involved limb. General examination revealed swelling of the left shoulder and a brachial pulse deficit at the left antecubital fossa and a radial pulse deficit at her left wrist. Neurological examination revealed complete loss of left wrist and hand power of 0/5 in all myotomes. Sensory examination revealed poor sensation in a median, ulnar and radial distribution. An X-ray was performed of her left shoulder which showed a fracture of the left proximal humerus with severe displacement of the humeral shaft into the axilla [Figure 1]. A CT scan of her left shoulder showed a two-part fracture of the left proximal humerus with subluxation of the head and medial displacement of the humeral shaft into the axilla [Figure 2]. The CTA of her left axillary artery showed lack of distal flow beyond the 2nd part of the axillary artery with poor collateral flow [Figure 3].

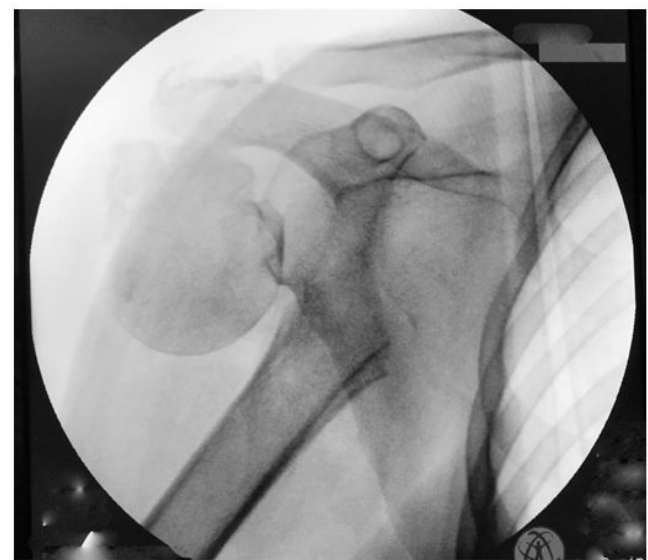

Figure 1: Shoulder X-ray: Fracture of the left proximal humerus with medial displacement of the shaft of the proximal humerus into the axilla. These are subluxation of the head of the humerus.

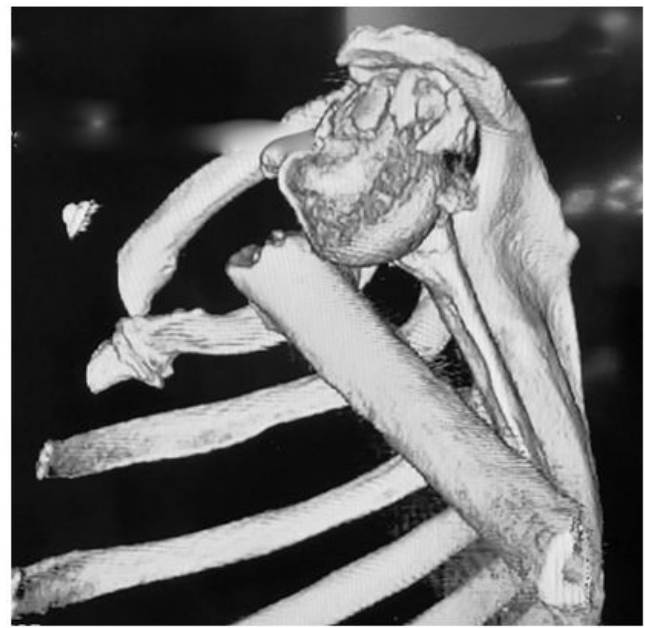

Figure 2: CT scan left shoulder lateral view: 3 D view of left shoulder showing displaced fracture of the left proximal humerus

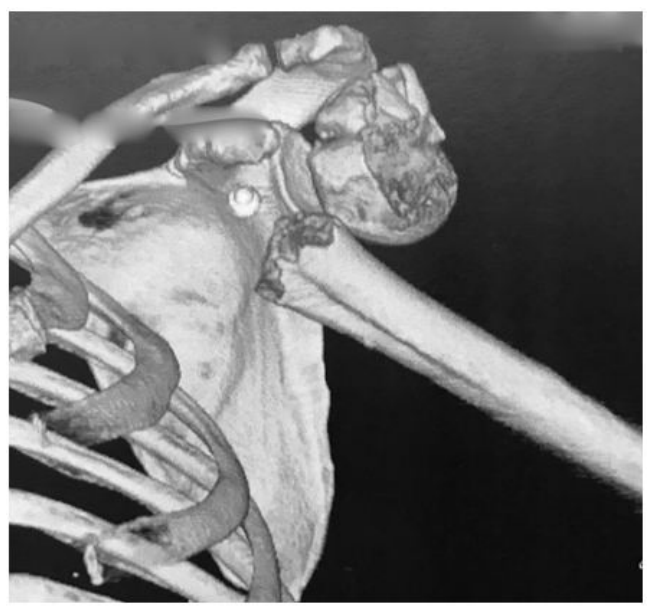

Figure 3: CT scan left shoulder anteroposterior view: $3 \mathrm{D}$ view of left shoulder showing medial displacement of the shaft of the proximal Humerus. There is subluxation of the head of the Humerus. 
She was booked as an emergency case and was operated under a general anaesthetic. No regional block was given due to the suspected brachial plexus injury. A delto-pectoral approach was utilized to access the fracture site. Open reduction and internal fixation of the left proximal humerus was performed for fracture stabilization. This was achieved with a humeral locking plate and screws [Figure 4]. Intra-operatively there was a deficit in the bone of the humeral head which was filled with allograft. Post fracture stabilization she was taken to the angiography suite where digital subtraction angiography confirmed an axillary artery intimal tear and a $4.5 \mathrm{~cm}$ dissection [Figure 5]. A digital subtraction angiographic interventional procedure was performed and a self-expanding endovascular stent was placed across the dissection [Figure 6]. The control angiography showed normal outflow

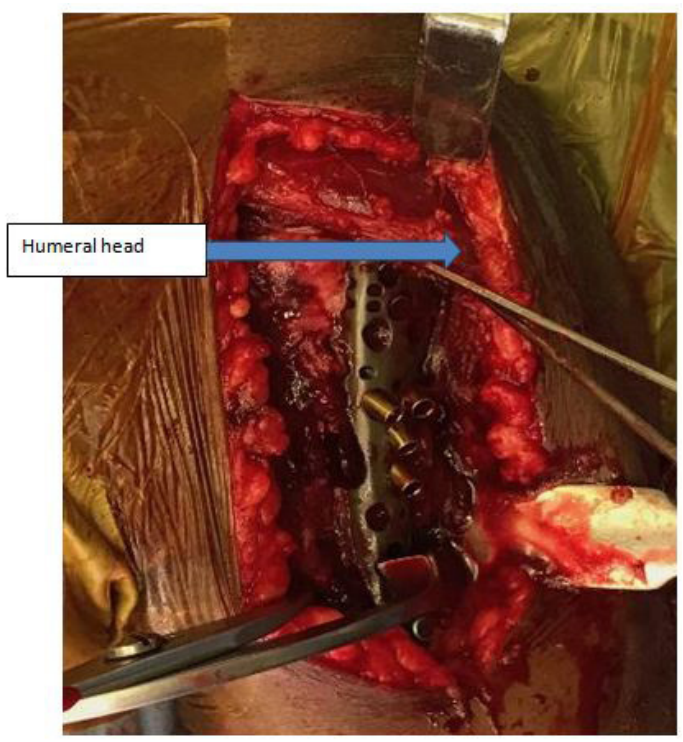

Figure 4: Intra-operative photograph: Showing deltopectoral approach with locking plate and screws being inserted to reduce the fracture.

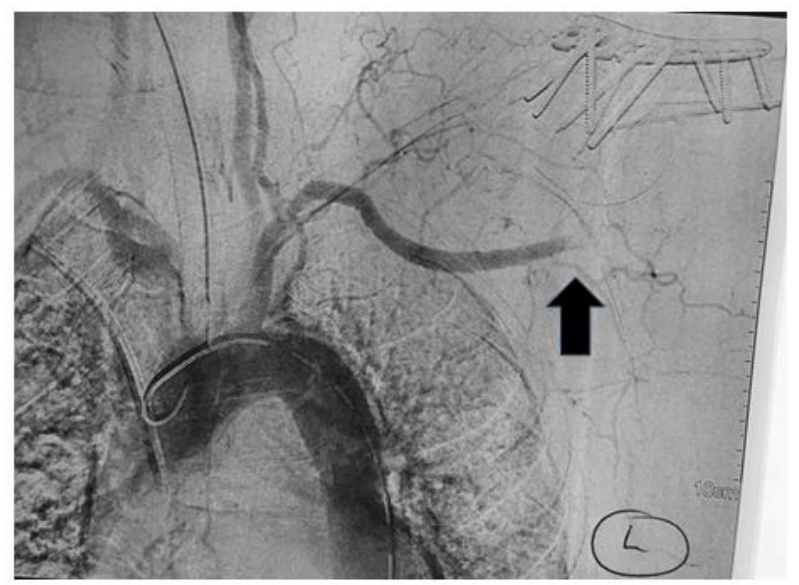

Figure 5: Digital subtraction angiography with arrow indicating site of occlusion of the left axillary artery with no distal flow and poor collateral circulation.
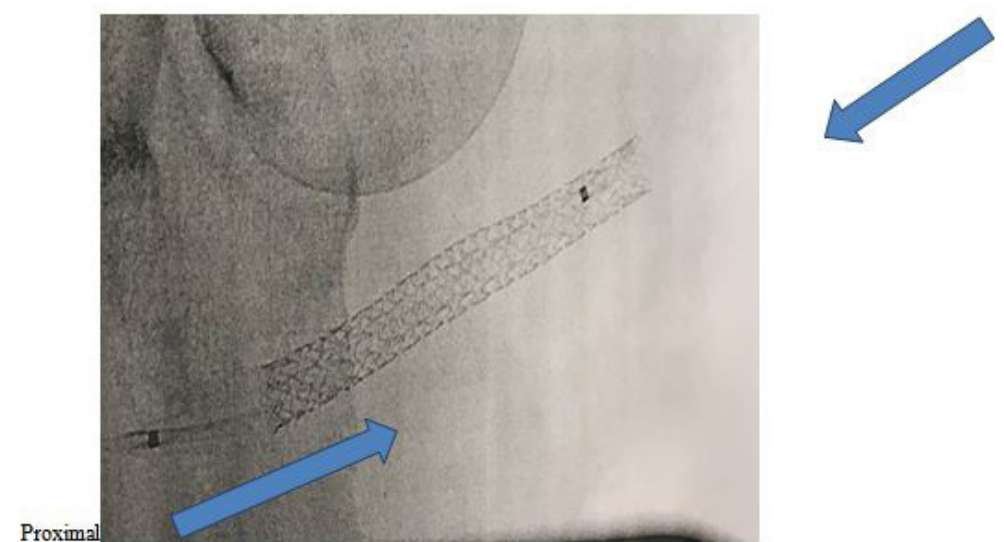

Figure 6: $\mathrm{X}$ ray: Self expanding stent in the left axillary artery 
of the axillary artery with good distal brachial artery filling. Restoring distal circulation was the priority in this case and the interval from the patient's initial fall until restoration of circulation in the angiography suite was four hours and fifty minutes. She was transferred to the Intensive care unit for observation and pulse checks and was commenced on enoxheparin 40IU injected subcutaneously twice daily and rivaroxaban $15 \mathrm{mg}$ orally daily. In the Intensive care unit her arm was supported in a sling and she received physiotherapy. In the immediate post-operative period clinical examination and vascular imaging studies confirmed good flow distal to the injured arterial segment [Figure 7]. There was however a two week delay before any improvement of motor function and hence the presence of concurrent brachial plexus palsy was suspected which was confirmed to be a neuropraxia on nerve conductive studies. This was managed conservatively and gradually improved over the subsequent weeks.

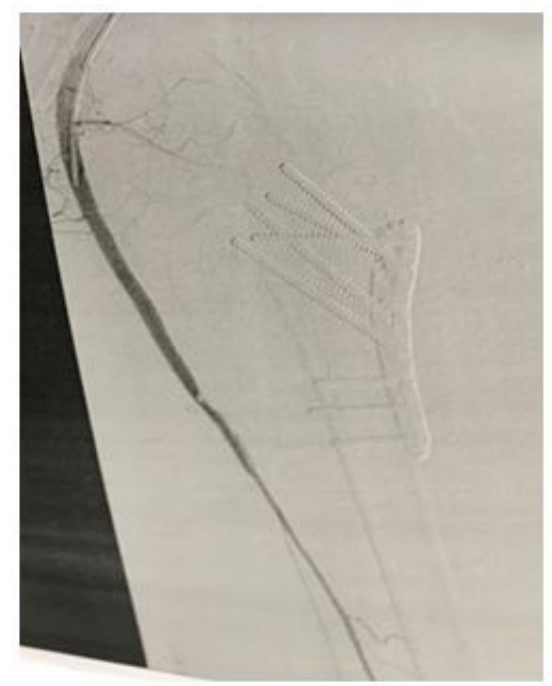

Figure 7: Digital subtraction angiography: showing restoration of distal flow in the left brachial artery post stent expansion.

At her three month out-patient review the patient was noted to have a moderately restricted range of motion of her left shoulder despite receiving ongoing out-patient physiotherapy. The power in her left elbow, wrist and hand had significantly improved to $3 / 5$ in all myotomes and she was able to feed herself with the injured limb as well as perform her activities of daily living with symptoms. She was pleased with the result and declined further operative intervention.

\section{Discussion}

Displaced fractures of the proximal humerus causing an axillary artery injury and brachial plexus palsy are rarely seen and are commonly reported in the literature as isolated case reports or as a short series with low patient number [1-4]. Due to tethering from the anterior and posterior circumflex arteries the axillary artery finds itself vulnerable to compression by the humeral shaft once severe displacement of the humeral shaft occurs [3]. This axillary artery injury is commonly blunt in nature due to the protection afforded by the surrounding fascial sheath [2]. The classical signs of a closed vascular injury include a reduced or pulse less extremity that is cold, pale with poor capillary refill in the affected vascular distribution.An expanding hematoma is rarely seen in these cases as the injury is commonly blunt in nature and confined by the surrounding fascial layers [2].With severe displacement combined with these signs an axillary artery injury should be suspected and a CT angiogram or digital subtraction angiography performed [5].

Looking at the association between blunt axillary artery injury and shoulder trauma it is noted that the majority of blunt axillary artery injuries occur with anterior gleno humeral dislocations. A lesser amount is due to proximal humeral fractures and the least amount is due to fracture dislocations of the humerus [6,7]. Prolonged ischaemia of the limb may result in necrosis of the hand and may even result in amputation. McLaughlin et al reviewed 19 proximal humeral fractures that had an associated axillary artery injury and in their study a $21 \%$ amputation rate was noted. In this study $53 \%$ of cases had an associated brachial plexus injury[8]. A brachial plexus injury presenting as a motor or sensory deficit should hence be interpreted as a marker of the probability that an axillary artery injury has occurred even in a patient with no acute clinical signs of limb ischaemia [8].

Various factors have been proposed as red flags to alert the attending surgeon to the possibility of an axillary artery injury in association with a proximal humeral fracture and include advanced age, atherosclerosis and osteoporosis [9]. The mechanisms by which the axillary artery injury occurs have been described and include hematoma of the arterial wall and secondary arterial dissection as occurred in our case, avulsion or rupture of the axillary artery due to overstretching, or direct laceration by the fracture fragments [3,9].

Diagnosing axillary arterial injury is often difficult as the distal pulses may be preserved in the context of adequate collateral flow [10]. Kelley et al described the triad of (1) shoulder trauma, (2) reduced distal pulses and (3) an expanding axillary mass as 
pathognomonic of an axillary artery injury [9]. While this triad may be useful in the context of gross full thickness injury to the vessel wall, lesser degree injuries such as intimal tears may present in a delayed fashion and in fact acutely the patient will have normal distal pulses and no axillary mass as no active bleeding occurs $[4,10]$. This scenario is further complicated by adequate collateral flow which makes diagnosis in the acute setting extremely difficult [10].A high index of suspicion needs to be in place for axillary artery injury in severely displaced proximal humeral fractures and frequent pulse checks instituted. A CT angiogram or even digital subtraction angiography should be used to confirm or exclude this vascular injury if any concern regarding a potential vascular injury presents itself [5]. Parasthesias rather than a pulse deficit are the most useful symptom that should alert the attending surgeon to the possibility of an axillary artery injury [11].

Once diagnosis of an axillary artery injury has been made the critical consideration is the interval between injury and repair. The critical time period for repair of axillary artery injuries is 4 hours for proximal injuries and 12 hours for distal injuries [12].

Displaced proximal humeral fractures with an associated axillary artery injury and brachial plexus palsy are increasing being diagnosed. It is unclear however if this is a true rise in incidence or simply due to increased in-patient admissions for proximal humeral fractures. It may simply be that we are detecting more axillary arterial injuries in association with this fracture pattern than was done in the past [13].

In the opinion of the authors the initiating event in this case was the humeral fracture displacement which happened at the moment of impact. The axillary artery injury similarly occurred at the moment of impact but due to the injury being contained within the facial sheath there was no frank axillary mass/ hematoma at presentation. Over several minutes to an hour the injured axillary artery became swollen with clot contained within the wall of the vessel. This would ultimately present angiographic ally as the dissecting aneurysm. The enlarging swollen axillary artery then began to exert pressure on an already stretched brachial plexus secondary to the humeral shaft displacement. Due to the injured axillary artery and brachial plexus being contained within the same facial sheath the pressure of the expanding axillary artery was exerted directly onto the brachial plexus causing pressure on the vasa nervorium which course along the epineurium and supply the nerve with blood. The brachial plexus was hence rendered ischemic which resulted in the brachial plexus palsy.

\section{Conclusion}

We report a rare case of a severely displaced fracture of the proximal humeral shaft that caused blunt trauma to the axillary artery and brachial plexus palsy. Fortunately a high index of suspicion and immediate surgical intervention by the attending orthopedic, interventional vascular surgeon and neurosurgeon prevented major complications.

We recommend a high index of suspicion for an associated axillary artery injury in the context of a severely displaced humeral fracture based on the presence of neurological symptoms rather than the classical signs of a vascular injury.

\section{References}

1.Modi CS, Nnene CO, Godsiff SP, Esler CAN (2008) Axillary Artery Injury Secondary to Displaced Proximal Humeral Fractures: A Report of Two Cases Journal of Orthopedic surgery. J Orthop Surgery 16: 234-46.

2.Hofman M, Grommes J, Krombach GA, Schmidt-Rohlfing B (2011) Vascular injury accompanying proximal humeral fractures: two cases and review of the literature. Emerg Med Int 2011: 742870.

3. Theodorides T, de Keizer C (1976) Injuries of the axillary artery caused by fractures of the neck of the humerus. Injury 8:120-3.

4. Hayes JM, Van Winkle G (1983) Axillary artery injury with minimally displaced fracture of the neck of the humerus. J Trauma 23:431-3.

5. Yaguban M, Panneton JM (2004) Axillary artery injury from humeral neck fracture: a rare but disabling traumatic event. Vasc Endovascular Surg 38:175-84.

6.Creagh TA, BroePJ, Grace PA, Bouchier-Hayes DJ (1991) Blunt trauma-induced upper extremity vascular injuries. J R CollSurgEdinb 36:158-60.

7.Raskin KB (1993)Acute vascular injuries of the upper extremity. Hand Clin 1:115-30.

8. McLaughlin JA, Light R, Lustrin I (1998) Axillary artery injury as a complication of proximal humeral fractures. J Shoulder Elbow Surg 7:292-4.

9. Kelley SP, Hinsche PA, Hossain JF (2004) Axillary artery transection following anterior shoulder dislocation: classical presentation and current concepts. Injury 35:1128-32.

10.Jensen BV, Jacobsen J, Andreasen H (1987) Late appearance of arterial injury caused by fracture of the neck of the humerus. J Trauma 27: 1368-9.

11. Zuckerman JD, Flugstad DL, Teitz CC, King HA (1984) Axillary artery injury as a complication of proximal humeral fractures. Two case reports and a review of the literature. ClinOrthopRelat Res 189:234-7.

12. Murata K, Maeda M, Yoshida A, Yajima H, Okuchi K(2008) Axillary artery injury combined with delayed brachial plexus palsy due to compressive hematoma in a young patient: a case report. J Brachial PlexPeripher Nerve Inj 3: 1-5.

13. Menendez ME, Ring D, Heng M (2015) Proximal humerus fracture with injury to the axillary artery: a population based study. Injury 46:1367-71. 


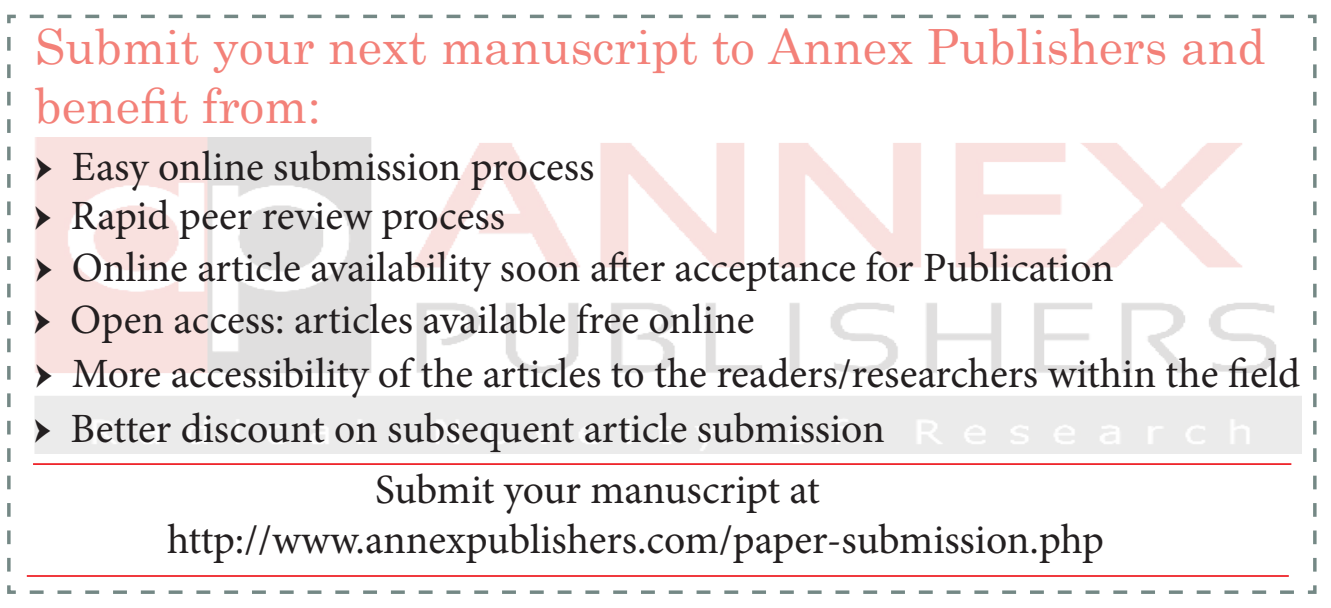

